\title{
Plenary lectures
}

\section{DISCUSSION OF NANOSCALE FRICTION - LUBRICATION EFFECTS ON MODEL ARTICULATING SYSTEMS}

\author{
P. Bełdowski ${ }^{1}$, A. Gadomski ${ }^{1}$, I. Wilczyńska ${ }^{1}$, \\ W. K. Augé II ${ }^{2}$ \\ 1University of Technology and Life Sciences, Institute of \\ Mathematics and Physics, PL - 85796 Bydgoszcz, Poland \\ 2 Department of Research and Development, NuOrtho Surgical, \\ Inc., at the Advanced Technology \& Manufacturing Center, \\ University of Massachusetts Dartmouth, Fall River, MA 02723 \\ USA
}

The disease burden associated with articular cartilage damage has encouraged the development of new techniques for synovial joint therapeutics. To develop such techniques, it is important to understand mechanisms governing natural synovial joint function. We present a facilitated lubrication model of articular cartilage (AC) which includes an autonomous threedimensional deterministic system of ordinary firstorder differential equations to describe the kinetics of the tribomicellization process, occurring with the aid of synovial fluid, and multilayer lipid membranes lining the articulating surfaces. This model assumes linear coupling of the material surface dislocation density and polyreaction fields and is in full agreement with lamellar-roller-bearing mechanisms which result in lower friction-coefficient values. This facilitated lubrication is achieved due to the bearing effects assured by micellar and other amphiphilic aggregates emerging at the articulation interface. Despite a mesoscale view, the model also predicts nanoscale effects that appear as a proton flux in quasiion channels (proton channels) [1, 2]. Such a phenomenon is consistent with treatment techniques which use proton flux effects in AC systems [3]. By deploying in situ electromagnetic field quanta during synovial fluid media replacement, one may achieve various beneficial therapeutic effects induced by proton motion. For instance, through biomimic engineering, the early surgical nanoscale tissue rescue treatment of $\mathrm{AC}$ damage with motive proton delivery gradients generated by physiochemical scalpels have been designed from alternating current redox magnetohydrodynamic technology [3]. These energybased surgical devices use inverse mass ratio batteries, an advance in energy production that deploys an "irrigant within water" designed from the scientific discipline of engineered irrigants. The milieus in which the scalpels have to operate possess their own acid-base equilibrium, resulting in some $\mathrm{pH}$ characteristic value. The scalpels, when intruding them, perturb this equilibrium, presumably in favor of employing the proton electromotive gradients. They are coupled, however, with the acid-base milieus' characteristics, causing some essential changes to occur, especially when they override their ranges of action. Moreover, the milieu per se is an anomalously relaxing soft material, very responsive to even minor deficits in its biochemical content [4]. The basic physiologic features are modified, even considerably, as it is expected to occur in (extra) cellular matrices of animals [5] or those of vacuoles-involving plants [6].

Acknowledgments: This work is supported by the project BS5/2009 at UTP Bydgoszcz.

[1] Gadomski A., Pawlak Z., Oloyede A. (2008) Directed ion transport as virtual cause of some facilitated friction lubrication mechanism prevailing in articular cartilage: a hypothesis, Tribol Lett 30: 83.

[2] Gadomski A., Bełdowski P., Rubi J. M., Urbaniak W., Augé II W. K., Santamaria - Holek I., Pawlak Z., Some conceptual thoughts toward nanoscale oriented friction in a model of articular cartilage, Math. Biosci., DOI 10.1016/j. mbs. 2013.05.004.

[3] Augé W. K. (2012) Inverse mass ratio batteries: An in situ energy source generated from motive proton delivery gradients, Nano Energy 309.

[4] Wilczyńska I., MSc Thesis, University of Technology and Life Sciences, Bydgoszcz 2012.

[5] Decoursey D. E. (2003) Voltage - gated proton channels and other proton transfer pathways, Physiol. Rev. 83:475.

[6] Barkla B. J., Pantoja O. (1996) Physiology of ion transport across the tonoplast of higher plants, Ann Rev Plant Physiol Plant Mol Biol 47:159.

\section{THE TERAHERTZELECTROMAGNETIC RADIATION: FEATURES OF NONTHERMAL CONVERSION INTO OTHER TYPES OF ENERGY}

\section{Yu. P. Chukova}

The Moscow Society of researchers of nature, Krasnopresnenskiy Ecological Fund. Moscow, Russia

Now terahertz electromagnetic waves are used in experimental biology and medicine. Unlike the experiment which history only begins, the theory of efficiency of conversion of such radiation into other kinds of energy has been developed in $70-s^{\prime}$ years and published in Russian scientific editions. The paper [1] was published also in a translation into English. The received results interested nobody for a long time, 
but now they are capable to help to understand the current and future experiments.

As it has been shown by thermodynamic method [2, $3]$, in the Wien region (hv>>kT) efficiency of conversion of energy of electromagnetic radiation into other kinds of energy submits to the Weber-Fechner law, and in the Rayleigh-Jeans region $(\mathrm{h} v<<\mathrm{kT})-$ to the Devyatkov law. These laws differ from each other considerably, and terahertz radiation lays in an interval between them. It is shown, how there is a transition from the Weber - Fechner law to the Devyatkov law. Difficulties of experiments under terahertz radiation are discussed. Division of effects on thermal and nonthermal ones in this area will have difficulties.

[1] Chukova Yu. P. (1977) The maximum efficiency for the conversion of light energy to chemical energy Khim Vys. Energ., 11, 126 - 131 [High Energy Chem. 1977, 11, 100 $-104]$.

[2] Chukova Yu. P. Advances in nonequilibrium thermodynamics of the systems under electromagnetic radiation. Khrizostom, Moscow, 2001, 118 p., ISBN 5-7508-9285-X.

[3] Chukova Yu. P. The Weber - Fechner law. To the $150^{\text {th }}$ anniversary of the publication of the book "Elemente der Psychophysik" by G.T. Fechner. 2010, Moscow, ZAO MP "Gigiena" 180 p. ISBN 978-5-904207-07-6.

\section{EMERGENCE OF SELFORGANIZATION IN AQUEOUS SYSTEMS AND LIVING MATTER}

\section{E. del Giudice ${ }^{1}$, P. Stefanini ${ }^{2}$}

\footnotetext{
${ }^{1}$ Centro Studi Eva Reich Via Orti, 5, 20122 Milano, Italy

${ }^{2}$ Nextcare s. a. s Via di Villa Barberino, 3, 50026 San Casciano (Firenze), Italy
}

The ability to self-organize is the main feature of living systems. This ability is the consequence of the spontaneous decrease of entropy in living systems, as pointed out by Schroedinger and Prigogine. This property occurs in quantum systems when coherence, namely the appearance of a well defined value of the phase, gets established. The appearance of this property demands the release of energy which can occur when the system is open. In aqueous systems a hierarchical structure of nested coherent systems is generated providing an ensemble of correlated frequencies, whose ensemble gives rise to a fractal music, which has been experimentally detected. The same property occurs in living systems, whose main component is just water. This music could be the physical basis underlying psychical phenomena, accounting for the close connection between psyche and soma observed in a number of therapeutic approaches.

\section{BIOLOGY O THE THRESHOLD OF A QUANTUM REVOLUTION}

\author{
P. Davies ${ }^{1}$, L. A. Demetrius ${ }^{1,3}$, J. A. Tuszynski ${ }^{4,5}$
}

${ }^{1}$ Beyond Center for Fundamental Concepts in Science, Arizona State University, Tempe, Arizona, 85287-1504, USA

${ }^{2}$ Department of Organismic and Evolutionary Biology, Harvard University, Cambridge, Massachusetts, 02138, USA

${ }^{\mathrm{c}}$ Max Planck Institute for Molecular Genetics, Berlin, Germany

${ }^{3}$ Department of Oncology, University of Alberta, Edmonton, Alberta, T6G 1Z2, Canada

${ }^{4}$ Department of Physics, University of Alberta, Edmonton, Alberta, T6G 1Z2, Canada

This talk reviews the role of the Planck constant in physics starting from its intoduction to describe black body radiation, its crucial role in the Schroedinger equation and the Gaussian wavepacket spreading. More formally, the Planck constant plays a defining role in the Heisenberg uncertainty principle and appears in the Einstein-Debye statistics of solids. Quantum effects in biology are discussed and include biophotons, centriole functions, Froehlich's coherence, visual perception, olfactory sensing, bird navigation, light harvesting and photosynthesis as well as quantum evolution, bioenergetics and quantum metabolism. The latter problem leads to the definition of a biological equivalent of the Planck constant. We then elaborate on its significance in the context of the uncertainty principle: time-energy, positionmomentum, and the length, time and energy scales, involved in the ATP production in mitochondria. It is concluded that the eleven orders of magnitude (from $10^{-34}$ to $10^{-23}$ ) between physical and biological Planck constants correspond to the scale gap between physical building blocks of matter: elementary particles and biological functional units: living cells.

\section{BIOLOGICAL COHERENCE AS A BASIC CONDITION FOR COGNITION AND INTELLIGENT BEHAVIOR}

\author{
I. Jerman ${ }^{1}$, M. Plankar ${ }^{1}$, S. Brežan ${ }^{2}$ \\ ${ }^{1}$ Institute Bion, Stegne 21, 1000 Ljubljana, Slovenia \\ ${ }^{2}$ (Independent researcher), Adamičeva 1, 1230 Domžale, Ljubljana, \\ Slovenia
}

Coherence has been substantiated as an autonomous and fundamental mechanism of biological selforganization due to its capacity for an efficient energy transfer and information processing. Coherent phenomena do not only pertain to molecular dynamics (e.g., the recently emerging quantum biology), but operate at all levels of biological organization, giving rise to synchronized metabolic cycles, various 
physiological rhythms and collective dynamics within large groups of organisms (Plankar et al 2011, 2012, $2013 \mathrm{a}, \mathrm{b})$. It is of particular interest whether and how quantum and electromagnetic dynamics at the molecular scale couples to and influences macroscopic biological processes. We suggest that coherence may represent a functional principle of such coupling, thus promoting complex, yet highly organized macroscopic behavior of biological systems.

The overall importance and hierarchical organization of biological coherence comes especially to the forefront in the brain dynamics. It is known that coherent oscillations of large neuronal assemblies (which are basically electromagnetic events, measured e.g. as electric potential fluctuations via EEG) in various frequency bands and in various brain regions, correlate with all basic cognitive functions (perception, attention, memory, decision making and consciousness), mediate local and long-range neuronal communication, and affect synaptic plasticity. In mediating cognitive functions, coherence does not only promote an efficient information transfer by input summation, it also acts as an information coding system that is based on phase and frequency of neuronal oscillations (Fries 2009). Specifically, many psycho-neurophysiological studies have shown explicit correlations between levels/characteristics of intelligence parameters and distinct coherence (interregional synchrony or phase consistency/ delay dynamics) and/or amplitude/ power (local (de)synchronization) patterns of human brain oscillations in different frequency bands (Thatcher RW, 2005; Jaušovec, 2005; Langer et al., 2012). An increased neural efficiency and higher brain complexity was predicted to be positively related to intelligence (Anokhin et al., 1999, Jausovec and Jausovec, 2003), therefore in such context negative correlations between widespread-scale EEG coherence and IQ are expected, together with more selective involvement of cortical areas with increased coherence, altogether reflecting increased spatial differentiation and complexity of the brain, enabling increased speed and efficiency of information processing (Silberstein, 2004; Jausovec, 2000).

However, it seems that certain patterns of extremely fast and complex oscillatory changes observed experimentally could not be sufficiently explained exclusively on the basis of the well established synaptic mechanisms, which indicates that a deeper understanding is needed (Freeman and Vitiello 2006). On the other line of research, theoretical modeling as well as the recently emerging experimental evidence indicates that microtubules support highly cooperative energy transport and information processing that is based on coherence. A growing body of research further indicates that the intraneuronal matrix, composed of cytoskeletal elements and their binding proteins, as well as up - to - now much ignored extracellular matrix, seem to be involved in neuronal signal integration via electric signaling due to their charged surface. This highly interconnected system of filamentous matrices may thus act as a 'bridge' between micro and macro levels of life, in other words, between quantum and classical mechanisms of information processing.

Coherence could thus represent a common denominator of neurophysiological and biophysical brain processes, operating at multiple levels of neuronal organization, from which cognition and animal/human intelligence may emerge as its cardinal manifestation. In this connection it is interesting that the anatomy of primate (mammalian) brain is quite different from the one of birds, yet both groups of organisms may demonstrate strikingly similar intelligent behavior. Therefore, if we follow what we learned from the brain dynamics, intelligence and cognition, demand a highly organized coherent, i.e., deeply integrated, innerly connected, system. Thus biological coherence can well be at the basis also of non-brain phenomena of intelligent (purposeful) behavior that was found not only in lower animals but also in plants and microorganisms (Trewavas 2005; Trewavas, Baluška 2011).

[1] Anokhin A. P., Lutzenberger W., Birbaumer N., (1999) Spatiotemporal organization of brain dynamics and intelligence: an EEG study in adolescents, Int $J$ Psychophysiol; 33(3):259- 73 .

[2] Freeman W. J., Vitiello, G. (2006) Nonlinear brain dynamics as macroscopic manifestation of underlying many-body field dynamics Physics of life reviews, 3(2):93 $-118$.

[3] Fries P. (2009) Neuronal gamma-band synchronization as a fundamental process in cortical computation Annual Review of Neuroscience, 32:209 - 224.

[4] Jausovec N., Jausovec K. (2003) Spatiotemporal brain activity related to intelligence: a low resolution brain electromagnetic tomography study, Brain Res Cogn Brain Res, 16(2): $267-72$.

[5] Jaušovec N., Jaušovec, K. (2005) Differences in induced gamma and upper alpha oscillations in the human brain related to verbal/performance and emotional intelligence, International Journal of Psychophysiology, 56(3): 223 235.

[6] Jaušovec N. (2000) Differences in cognitive processes between gifted, intelligent, creative, and average individuals while solving complex problems: an EEG study, Intelligence, 28(3): $213-237$.

[7] Langer N., Pedroni A., Gianotti L. R., Hänggi J., Knoch,D., Jäncke, L. (2012) Functional brain network efficiency predicts intelligence, Human brain mapping, 3(6):1393 - 1406.

[8] Plankar M. (2013b) Coherence in biological systems and its applications to life sciences. Doctoral dissertation. University of Ljubljana, Biotechnical faculty, pp. 113.

[9] Plankar M., Brežan S. in Jerman, J. (2013a) The principle of coherence in multi - level brain information processing. Progress in Biophysics \& Molecular Biology, 111: $8-29$.

[10] Plankar M., Del Giudice E., Tedeschi A. in Jerman, I. (2012). The role of coherence in a systems view of cancer development. Theoretical Biology Forum, 105:15 - 46. 
[11] Plankar M., Jerman I. in Krašovec, R. (2011). On the origin of cancer: Can we ignore coherence? Progress in biophysics and molecular biology, 106(2): $380-390$.

[12] Silberstein R. B., Song J., Nunez P. L., Park W. (2004) Dynamic sculpting of brain functional connectivity is correlated with performance. Brain Topogr., 16(4):249 54.

[13] Thatcher R.W., North D., Biver C. (2005) EEG and intelligence: relations between EEG coherence, EEG phase delay and power, Clinical Neurophysiology, 116(9):2129-2141.

[14] Trewavas A. J. (2005) Plant intelligence, Naturwissenschaften 92: 401-413, DOI10.1007/s00114005-0014-9.

[15] Trewavas A. J., Baluška F. (2011) The ubiquity of consciousness, cognition and intelligence in life, EMBO reports Vol. 12, No. 12, pp. $1221-1225$.

\section{COCHLEAR IMPLANTS IN AN ELECTROMAGNETIC FIELD}

\begin{abstract}
M. Kordus
Department of Biophysics, Poznan University of Medical Sciences, Poland

Magnetic resonance imaging (MRI) is a widely performed medical imaging technique allowing the viewing of detailed internal body structures. It is utilized to provide the information required for diagnostic purposes by detecting various conditions. It has been shown that specific protocols need to be followed in order to safely perform MRI examinations in patients with cochlear implants. Notably, some devices require the use of MRI system not exceeding 0.2- or 0.3-Tesla as part of the protocol. In other cases, the magnet associated with the cochlear implant requires removal prior to the MRI examination. Studies indicate that electromagnetic fields created during MRI scan interfere with magnetic fields of the cochlear implant magnet causing its demagnetization and the appearance of torque phenomena in implanted devices.
\end{abstract}

[1] Dubrulle F., Sufana Iancu A., Vincent C., et al (2013) Cochlear implant with a non-removable magnet: preliminary research at 3-T MRI, Eur Radiol 25: 15101518.

[2] Jeon J. H., Bae M. R., Chang J. W., et al (2012) Reversing the polarity of a cochlear implant magnet after magnetic resonance imaging. Auris Nasus Larynx 39: 415-417.

[3] Dubrulle F., Vincent C. Varoquaux A., et al (2011) Guidelines for the performance of MRI in patients with cochlear implants. J. Radiol 92: 872-877.

[4] Caranne B. T., Gottschalk B., Kraut M., et al (2010) Magnetic resonance imaging at $1.5 \mathrm{~T}$ after cochlear implantation. Oto Neurotol 31: 1215-1220.

[5] Majdani O., Leinung M., Rau T., et al (2008) Demagnetization of cochlear implants and temperature changes in 3.0 T MRI enviroment. Otolaryngology Hen Neck Surg 139: 833-839.

\section{DOUBTER'S GUIDE TO THE BIOELECTROMAGNETISM}

\author{
O. Kučera ${ }^{1}$, D. Havelka ${ }^{1,2}$, M. Cifra ${ }^{1}$
}

${ }^{1}$ Institute of Photonics and Electronics, Academy of Sciences of the Czech Republic, Prague, Czechia,

${ }^{2}$ Department of Electromagnetic Field, Faculty of Electrical

Engineering, Czech Technical University in Prague, Prague, Czechia

The bioelectromagnetism, i.e. electromagnetic activity of living cells, is highly controversial topic because literally decades of intensive research on this topic have not brought defensible experimental results. With the exception of well - established electrophysiology and auto-luminescence, there are no trustful experimental data showing electromagnetic activity of cells. For frequencies between few hundreds of $\mathrm{Hz}$ and hundreds of $\mathrm{THz}$ we do not even have experimentally well - established indication that cells may generate electromagnetic field significantly higher than that of thermal noise. Despite this fact, large number of theories has emerged concerning generation of electromagnetic field in cells and also employment of this hypothetical field by cells.

Beside hypotheses which are quite far fetched, there are, of course, solid theories based on widely accepted physical mechanisms. However, when we put numbers in these theories we find that intensities of generated field are very low or rapidly decreasing with the distance from generating structure. Therefore, the relevance of direct force effects of such field in biological processes, if any, is very likely limited to very local area.

In our contribution, we illustrate this fact in silico on the vibrations of microtubules. Our results show that mechanical vibrations of microtubules should indeed generate electromagnetic field, but this field may have some translational or rotational force effects on particles/molecules only in the immediate vicinity of the microtubule. Assumption of the role of this field anywhere else than along the microtubule is hardly explainable by direct force effects. We predicted theoretically that the multimode excitation causes formation of electric wave packet and formation of many transient local electric field minima. This enables ultra-fast signal transfer along the microtubule lattice, which is conceptually more realistic.

We also critically discuss the possibility of cell - to - cell communication using electromagnetic waves. The motivation for us was that during decades a large number of experimental reports ascribing signaling between distant cell cultures to electromagnetic phenomena has appeared. However, rigorous analysis on this topic - both qualitative and quantitative - has never been published so far to our knowledge. Our results indicate that the electromagnetic communication between cells on a distance more than few micrometers could, as a general phenomenon, 
take place only under very special circumstances or using mechanisms not known to be present in cells.

In conclusion, we argue that the relevance of electromagnetic activity of living cells to physiology seems overestimated in the majority of suggested theories. Rather than speculating about endogenous electromagnetic waves on the level of cells and tissues and their hypothetical role in physiology, which seems to be far fetched based on the current knowledge, we encourage first to research local electromagnetic events involving molecules and macromolecules to provide solid foundations for electromagnetic phenomena on larger scale. Local events fit better to current state of knowledge in biophysics and they are also better candidates for experimental testing and potential applications. We have proposed one example: signal transfer along microtubule by electroacoustic wave.

\section{THEORETICAL STUDIES ON THE INTERACTIONS OF CYTOSTATIC PYRAZOLE DERIVATIVE WITH DNA BASES}

\section{J. Kujawski' ${ }^{1}$, M. Doskocz ${ }^{2,}$, B. Drabińska1, A. Myka1, J. Kruk'1, M. K. Bernard1}

${ }^{1}$ Department of Organic Chemistry, Poznan University of Medical Sciences, Poznan, Poland,

${ }^{2}$ Foundation for Development of Science and Business on Medical and Exact Sciences Area, Wroclaw, Poland,

3 RootInnovation sp. z o.o., Wroclaw, Poland

In a continuation of our previous study concerning the proapoptotic activity of several fused pyrazole derivatives [1], we investigated a possibility of the adhesive interactions between one of the above compounds, namely 5(3,5-dimethyl-1H-pyrazol-1-yl)3-[(4-methylphenyl)sulfonyl]-1H-indazole (1), and nucleosides. Although we suggested a likely mechanism of the cytotoxic action, the substance target at the molecular level is still unclear. Our earlier study showed that compound $\mathbf{1}$ was able to form strong hydrogen bonds through the pyrrolic nitrogen ( $\mathrm{NH}$, donor) and weak hydrogen bonds through the sulfonyl oxygens (acceptors) [2].

The interactions of compound $\mathbf{1}$ with adenosine, guanosine, cytosine, and thymidine as well as with a DNA fragment were investigated using a solvation sphere model. We observed that guanosine and pyrazole derivative 1 were linked through two strong hydrogen bonds $\mathrm{NH}^{\cdots} \mathrm{N}$. The bond energy equalled $-13.4 \mathrm{kcal} / \mathrm{mol}$ considering the BSSE error. The system stability as a box with 1049 water molecules (box diameter: $31.7 \AA$ ) was confirmed using molecular mechanics.

The theoretical analysis of the interactions between compound 1 and adenosine was carried out using three different models. The analysis showed the presence of $\mathrm{NH}^{\cdots} \mathrm{N}$ hydrogen bonding between the indazole moiety and the base, as well as $\mathrm{CH}^{\cdots} \mathrm{N}$ bonding between the benzene ring of indazole and adenosine and $\mathrm{NH}^{\cdots} \mathrm{O}$ bonds involving the sulfonyl group and adenosine. The calculated energies were $-15.7,-6.2$, and $-5.2 \mathrm{kcal} / \mathrm{mol}$, respectively. The molecular dynamics modeling revealed that in two of the three models the indazole and base rings were nearly coplanar. The box sizes and water content for the individual models were: a) $33.8 \AA, 1281$ water molecules; b) $40.0 \AA$, 2109 water molecules, and c) $31.2 \AA, 1009$ water molecules. Changes in the dihedral angle between the $\mathrm{NH}$ indazole and $\mathrm{CH}$ adenosine planes resulted only in small alterations of the $\mathrm{N}^{\cdots} \mathrm{CH}$ distance. The smallest length of this bond was due to the close proximity of the tosyl residue and adenosine and was not a feature of the lowest energy conformer.

The DFT optimization of the adduct indazole derivative $\mathbf{1}$ and cytosine showed the presence of two strong hydrogen bonds $\mathrm{NH}^{\cdots} \mathrm{O}=\mathrm{C}$; their calculated energy was $-14.8 \mathrm{kcal} / \mathrm{mol}$, i.e. only about $1 \mathrm{kcal} / \mathrm{mol}$ less than for the similar adduct with adenosine. The indazole and cytosine rings were not coplanar due to the close proximity of two $\mathrm{sp}^{2}$ hybridized nitrogen atoms with charges of -0.258 and $-0.643 \mathrm{eV}$ that caused mutual repelling. The molecular mechanics revealed that the box size was $38.3 \AA$ and it contained 1851 water molecules.

Considering the above discussion, we can conclude that the cytotoxic activity of compound $\mathbf{1}$ at the molecular level may be connected with its ability to adhesion to DNA bases through strong and weak hydrogen bonding.

[1] Totoń T., E. Ignatowicz, M. K. Bernard, J. Kujawski, M. Rybczyńska (2013) J. Physiol. Pharmacol. 64(1): 115 123 and reference cited herein.

[2] Kujawski J., Doskocz M., Popielarska H., Myka A., Drabińska B., Kruk J., Bernard M. K. (2013) J. Mol. Struct. 1047: $292-301$.

\section{Acknowledgments}

This study was supported by SBN Grants No. 126/2012, 174/2013 (Poznan University of Medical Sciences) and Grant No. 502-14-03308417-08345 (Poznan University of Medical Sciences), as well as PCSS (Poznan Supercomputing Center) Grant No. 92/2011, and carried out in cooperation with the Research and Development Laboratory of RootInnovation, Inc. 


\section{APPLIED QUANTUM PHYSICS: PATHOLOGICAL BIOCHEMICAL PATWAYS CURED BY HIGH HOMEOPATHIC POTENCIES OF THEIR SUBSTRATES, INHIBITORS AND ENZYMES}

\author{
K. Lenger \\ Institute for Scientific Homeopathy, Kaiserstr. 28, \\ DE - 63065 Offenbach
}

Homeopathic treatment is performed according to the Law of Similars by using highly diluted and succussed homeopathic remedies without containing molecules any more. Up to now symptoms are cured by a homeopathic therapy without any control of laboratory values. Therefore homeopathy has great difficulties to be acknowledged.

Biochemical function of homeopathic remedies was only proposed by Schüßler's Biochemie using low homeopathic potencies of the body's minerals. Since Lenger detected photons with different frequencies in highly diluted and succussed remedies by two magnetic resonance methods, a scientific base of homeopathy is now thinkable. The Law of Similars can be expressed as: the frequencies of the patients must match those of the homeopathic remedies. In analogy to the experiments proving photons in homeopathic remedies it can be assumed that the remedies' photons are attracted by the stronger electromagnetic field of the biochemical pathological pathway having similar frequencies. Thereby the photons are separated from their carrier substances, ethanol or sugar. The ill making frequencies are then attenuated by resonance and the original biochemical pathway will be restored. Lenger used highly diluted and succussed reaction substrates, enzymes of the body and their inhibitors proved by chemical trials.

Pathological biochemical pathways can be indicated by different laboratory values: The lack of $\mathrm{Fe}^{++}$, Ferritin, hemoglobin can easily be measured. The corresponding symptoms are mostly described in the homeopathic symptom picture: a terrible whooping cough, asthma, weakness, faintness, cramps in the muscles, paralyses. During that sickness consciousness is altering, too. A group of 25 patients having these symptoms was treated in the following way: First, the respiration chain with its cytochrome systems $a$ and $b$ containing $\mathrm{Fe}^{++}$and $\mathrm{Cu}^{++}$has to be considered: Ferrum metallicum LMK, a high potency, given every day, heals the cough and the $\mathrm{Fe}^{++}$value is becoming normal within one month. This treatment was supported by Ferritin LMK. Ferritin is the iron storing protein in the liver. In case that the cough remained constant, the LMK-potency of hydrocyanicum acid or potassium cyanide potent lethal inhibitors of the $\mathrm{Fe}^{++}$-pathway had been added. They form hexacyanoferrate-complexes with $\mathrm{Fe}^{++}$, so that the iron cannot easily be proved chemically. This gives the impression that it is "lost".

In patients, the lack of iron can be caused by infection with Bordetella Pertussis, Haemophilus Influenzae, Chlamydiaceae or Hepatitis A, B, C. All of them use the iron for their own nourishment. So, iron is getting lost in the respiration chain, in ferritin, in hemoglobin. This state can be seen in homeopathy as if the iron had formed hexacyanoferrate-complexes. Highly potentized $\mathrm{HCN}$ or $\mathrm{KCN}$, together with Ferrum metallicum LMK and Ferritinum LMK, restored the iron in the respiration chain and normalized the iron, ferritin, and hemoglobin levels in the blood, proved by laboratory values. By this homeopathic treatment the described symptoms of more than 15 patients were cured within 6 weeks.

But the symptoms of 10 patients increased: asthma, aches in the abdomen, weakness, no energy in the muscles, paralyses nor the levels of $\mathrm{Fe}^{++}$, ferritin and hemoglobin did not remarkably increase. Now the therapy involving oxidative phosphorylation had to be considered. It involves the glycolysis, the citric acid cycle and the biosynthesis of the porphyrin system. Sometimes even porphyrin is measured in urine (Porphyria). In this case the formation of the porphyrin ring should be supported. That is the prosthetic group in the respiration chain as well as in hemoglobin.Therefore, intermediate substances of the glycolysis and/or the citric cycle had to be given in LMK-potencies daily. Since some of these substances are not available as homeopathic remedies such as glucose-6-phosphate, Glucosum LMK and Phosphorus LMK had been given instead. Adrenalinum and Insulinum in LMK known to stimulate the degradation of glucose had been added. Then Glycerinum, Pyruvicum acidum, CoenzymeA, Oxalaceticum acidum, Citricum acidum, Ketoglutaricum acidum, Succinicum acidum and Hemoglobinum all in LMK-potencies had been applied. Glutamicum acidum LMK instead of Glycinum not being available had been added to support the synthesis of the amino acid glycin, the nitrogen donor for the porphyrin ring.

All the remedies mentioned had been applied daily and together. After 4-6 weeks, the hemoglobin, $\mathrm{Fe}^{++}$ and ferritin levels of the 10 patients increased and the symptoms of the patients started to heal or vanished completely.

Five more patients suffering from paralyses additionally needed Arsenicum album LMK, Lachesis mutus LMK or Crotalus horridus LMK, the snake venoms. As allopathic - they are the inhibitors of the glycolysis, attacking its phosphate and $\mathrm{SH}$-groups of its enzymes. Which remedy of those has to be chosen depends on the individual symptoms of the patient.

For the first time it is shown that a homeopathic therapy can be performed reproducibly by applying high potencies of substrates, enzymes of the ill 
biochemical pathways, and by their inhibitors. Furthermore all that can be controlled by laboratoryvalues. Until healing was achieved the mentioned homeopathic remedies had to be given daily and simultaneously. This form of homeopathic therapy points the way to the future in order to cure chronical diseases.

[1] Lenger K. (2006) Homeopathic potencies identified by a new magnetic resonance method. Homeopathy - an energetic medicine. Subtle Energies and Energy Medicine 15(3):225 - 243.

[2] Lenger K., Bajpai R. P. (2008) Drexel M, Delayed luminescence of high homeopathic potencies on sugar globuli. Homeopath; 97:134-140.

[3] Lenger K. (2010) A new biochemical model of homeopathic efficacy in patients with chronic diseases. Subtle Energies \& Energy Medicine, 19(3):9 - 41.

\section{INFLUENCE OF MAGNETIC FIELD ON RHEOLOGICAL PROPERTIES OF BLOOD}

\section{A. Marcinkowska - Gapińska}

Rheological Laboratory, Department of Neurology, Karol Marcinkowski University of Medical Sciences in Poznan, Przybyszewskiego 49, 60 - 355 Poznan, Poland

Magnetostimulation is one of the methods used in physiotherapy, however not all mechanism of its action have been clarified so far [1]. The Viofor JPS instrument is applied in cases of pain related to arthrosis, migraine or other impairments in the organism, such as impaired bone healing, soft tissues healing, circulation impairment, stress $[1-4]$. Other studies related to the direct influence of magnetic field on blood and its rheological properties have also been carried out $[3,5-8]$. The aim of this study is the analysis of the effect of magnetic field on the rheological blood properties on the basis of my own research and literature data.

Blood viscosity measurements were carried out by means of an rotary-oscillatory rheometer Contraves LS40 performing both rotary measurements of the flow curve at descending shear rate $\gamma^{\prime}$ in the range from 100 to $0.01 \mathrm{~s}^{-1}$ within a 5 minute period and oscillatory measurements at a constant frequency $f=$ $0.5 \mathrm{~Hz}$ with descending shear amplitude $\gamma_{0}$ which allowed for estimation of the complex viscosity $\eta^{*}$ and its real and imaginary components, $\eta$ ' and $\eta$ ', respectively. Analysis of the influence of magnetic field on the hemorheological properties of blood was performed both in vivo and in vitro conditions. In the case of in vivo stimulation, blood samples were taken from patients suffering from pain who started the therapy with the use of the large applicator Viofor JPS and different programs "P" and applications "M" offered by the manufacturer with various magnetic field intensities. Each of patients donated blood samples twice: before and after the therapy. All blood and plasma viscosity measurements were performed at $37^{\circ} \mathrm{C}$. Rotary measurements were performed to obtain plasma viscosity and whole blood flow curves. For each blood sample hematocrit value was measured using the standard method. In the case of in vitro stimulation only blood samples were subjected to magnetic field stimulating applied by means of the small applicator (pillow) and using a single program and a single application method. The results obtained from the rotary measurements were analyzed by means of Quemada rheological model in order to obtain a quantitative description of red cells agreeability and deformability $[9,10]$. The parameters chosen for evaluation were: hematocrit value, plasma viscosity, whole blood viscosity at four chosen shear rates, Quemada model parameters: $k_{0}$ (measure of red cells agreeability), $k_{\infty}$ (measure of red cells stiffness) and $\gamma_{c}^{\prime}$ (measure of red cells tendency to aggregate), as well as the components of the complex viscosity $\eta^{*}$ : viscous $\left(\eta\right.$ ') and elastic $\left(\eta{ }^{\prime \prime}\right)$.

It was found that the influence of magnetic field on blood rheological parameters depended on the method and program of application. In order to perform a thorough statistical analysis, a larger group of patients would have to be studied by means of in vivo stimulation.

[1] Sieroń A., Zastosowanie pól magnetycznych w medycynie (red. Sieroń A.); $\alpha$-medica press; Bielsko Biała, 2002.

[2] Pecyna M. B., Wolnozmienne pola magnetyczne w badaniach psychofizjologicznych, Wydawnictwo Akademickie ,Żak”, Warszawa 2001.

[3] Kowal P., Marcinkowska - Gapińska A. (2005) Próba oceny wpływu terapii zmiennym polem magnetycznym $u$ pacjentów z chorobą naczyniową mózgu (An influence of the altered magnetic field on the hemorheological parameters in patients with cerebrovascular disease); Neuroskop 7: $135-138$.

[4] Pasek J., Mucha R., Gmyrek J., Sieroń A., Wpływ wolnozmiennego pola magnetycznego systemem Viofor JPS na zachowanie się parametrów ciśnienia tętniczego krwi osób z nadciśnieniem tętniczym, Balneologia Polska, 2006, tom XLVIII, nr 2, pp. 95 - 100.

[5] Dasdag S., Sert C., Akdag Z., Batun S. (2002) Effect of extremely low frequency electromagnetic Fields on hematologic and immunologic parameters in welders, Archives of Medical Research 33:29 - 32.

[6] Ciejka E., Gorąca A. (2007) Oddziaływanie pola magnetycznego o parametrach stosowanych w magnetoterapii na wybrane parametry biochemiczne krwi, Balneologia Polska, październik - grudzień, 234 - 242.

[7] Tao R., Huang K. (2011) Reducing blood viscosity with magnetic fields. Physical Rev. E, 84, $011905(5)$.

[8] Kowal P., Marcinkowska - Gapińska A. (2010), Analysis of the influence of magnetostimulation on the hemorheological parameters and on the result of thermographic examination; Some aspects of medical physics - in vivo and in vitro. Ed. by Zofia Drzyzga, 
Krzysztof Ślosarek. Olsztyn: HARD Publishing Company, $59-64$.

[9] Lerche D., Bäumler H., Kucera W., Meier W., Paulitschke M., Flow properties of blood and hemoreological methods of quantification. W Physical Characterization of Biological cells. Basic research and clinic relevance. Red. W. Scütt, H. Klinkmann, I. Lamprecht, T. Wilson, Verlag Gesundheit GmbH Berlin $1991,189-214$.

[10] Quemada D. (1981) A rheological model for studying the hematocrit dependence of red cell - red cell and red cell - and red cell - protein interactions in blood, Biorheology, 18:501 - 516

\section{THE MATHEMATIC OF BIOLOGICALLY EFFECTIVE EMFs: ARE MAXWELL'S, SCHRÖDINGER'S AND PAULI'S FORMALISMS COMPATIBILE AND COMPLETE?}

\section{W. H. Medinger}

\author{
International Institute for Research on Electromagnetic \\ Compatibility (IIREC), Ringstr. 64, \\ 3500 Krems an der Donau, Austria.
}

For the development of physical science (with biophysics being a segment thereof), the application of proper mathematical tools has had an enormous impact. In the theoretical elaboration of his concept of electromagnetism, Maxwell used Hamilton's quaternions which were a recent discovery in his days, but are replaced by the more convenient vector representation in contemporary physics. Schrödinger's famous equation is based on a complex valued wave function (with the correspondence principle of classical physical magnitudes and imaginary quantum operators being no way arbitrary). Pauli, in turn, introduced the matrices named after him as constituents of a 2-component wave function in a squared complex space in order to represent 2 -valued elctron spin. At that point, the circle is closed, for it turns out that, apart from a unity matrix, Pauli's matrices are the exact match of a matrix representation of quaternions. So, we notice that all these formalism are indeed compatible, but their formal variety remains unsatisfactory.

A most challenging issue in biophysics is the identification of the physical fields and/or potentials that provoke a biological response. It is not at all a matter of course that the fields handled in classical electrodynamic theory, and in electric engineering and radio technology as well (e.g. RF electric and magnetic force fields), are the true - or exclusive causes of biological impact. From the quantum perspective to biological systems which has a solid theoretical and experimental foundation now, it is evident that potentials, i.e. in the electromagnetic domain, the (scalar) electrostatic potential $\Phi$ and the magnetic vector potential $\boldsymbol{A}$, would govern the behaviour of the system. The physical significance of a difference in $\Phi$ is well known as voltage. Similarly, a descriptive notion was attributed to $\boldsymbol{A}$ by Maxwell, as the "electromagnetic momentum". Both terms ( $\Phi$ as the representation of electromagnetic energy, and $\boldsymbol{A}$ as the representation of electromagnetic momentum) are complementary and fundamental in terms of classical Hamiltonian theory. In the Hamiltonian operator (the quantum analogue to the classical energy term), the momentum is expanded to the so-called kinetic momentum including $q \boldsymbol{A}$, with electric charge $q$ as a scaling factor.

From the physical and biological principles considered so far, we conclude that biological systems are controled, in a fundamental way, by the physical quantities of electric charge, electric potential, electromagnetic momentum (i.e. magnetic vector potential), and electron spin.

Research conducted by Mae-Wan Ho revealed that exposition to the magnetic vector potential of toroidal coils with a negligible magnetic field rises abnormalities in pattern formation in Drosophila embryos, as well as exposition to solenoidal coils with a strong magnetic field would do, thus evidending the biological effectiveness of the vector potential $\boldsymbol{A}$. There is a need for more biological research replacing ordinary coils with toroids for magnetic field experiments.

According to accepted physical theory, electric and magnetic force fields acting on biological tissue emerge as the first derivatives of the potentials $\Phi$ and A. The Maxwell/Heaviside equations of classical electrodynamics are invariant with respect to a gauge transformation mediated by a scalar function $\chi$. The gauge conditions of classical electrodynamics level out a scalar derivative $S$ that emerges in a completed formalism applying Clifford's algebra which, in turn, proves to be the key to the unification (and simplification) of Maxwell's, Schrödinger's and Pauli's formalisms. This natural expansion of electrodynamics suggests that the scalar terms in the derivatives of electromagnetic potentials should be observable field effects, thus rendering expanded (or generalised) electrodynamics a testable theory.

For biological application, it seems interesting that a rapidly fluctuating source of high voltage, such as a pulsed power system, could induce noticeable scalar effects which explains extraordinary results of high energy impulse therapy, e.g. significant reduction of edema in acutely sprained ankles following only one treatment. Short-term magnetic pulses of 40-80 $\mathrm{mT}$ permeating the cell membrane obviously induce biological coherence which may be understood in the framework of Fröhlich's theory.

[1] Jung F. (2006). Geometrische Algebra und die Rolle des Clifford - Produkts in der Klassischen und Quantenmechanik. Diplomarbeit im Fachbereich Physik. 
Institut für Physik, Johannes-Gutenberg-Universität Mainz.

[2] Ho M. W., French A., Hafegee J., Saunders P. T. (1994). In: Ho M. W., Popp F-A., Warnke U. (Eds.). Bioelectrodynamics and Biocommunication (Singapore: World Scientific), p. 204.

[3] Pennington G. M., Danley D. L., Sumko M. H., Bucknell A., Nelson J. H. (1993) Pulsed, non-thermal, high frequency electromagnetic energy (DIAPULSE) in the treatment of grade I and grade II ankle sprains. Mil Med 158: $101-4$.

[4] Van Vlaenderen K. J. (2003) A generalization of classical electrodynamics for the prediction of scalar field effects. http://arxiv.org/abs/physics/0305098 [physics.class-ph].

\section{MORA-kHz RESONANCE DIAGNOSTICS USED FOR THE MEASURMENT OF THE NEED FOR HOMEOPATHIC DRUGS}

\section{K. P. Michalak}

Department of Biophysics, Poznan University of Medical Sciences, Poland

Homeopathy is the branch of medicine that is not commonly accepted due to the mechanism of interaction with the human body being not known in details. Since Lenger has detected frequency-specific photons in homeopathic drugs, the discussion concerning homeopathy has entered the new level. The main clinical idea of homeopathy is included in the sentence: "Similia Similibus Curantur" (SSC) which means that the symptoms of the overdose of the given drug are the same as the indications for its application.

One of the important problems in homeopathy is the choice of the proper drug and its dose that is necessary for the given patient. The symptoms are often indicated to be treated using different drugs, depending on the composed and complicated patient's interview, and the choice of the proper one is not a trivial task.

The new, simple, electronic method is presented that is probably able to support the choice of the drug and its dose. It is the MORA-kHz-resonance diagnostics. This method was developed by Franz Morrel and Erich Rasche. They have built the commercial device MORA (Med-Tronic GmbH, EN ISO 13485, EN ISO 9001). The idea of the method is the use of ampoules with a drug, amplifying the generated vibrations in $\mathrm{hKz}$ range and conducting these signals through the human body. These signals are characterized by the increase in the impedance of about $10 \%-30 \%$ if the pathology connected with drug deficiency exists in the organism. The human body can be treated as the frequency-specific filter for the applied signal. The impedance increase of the tissue is observed only up to the given amplification of the applied signal. The highest amplification of the given signal being damped by the body points to the amount of energy, the body is able to absorb. In the case of homeopathic drugs, it makes it possible to find out, which drug vibrations are damped / sucked most strongly by the human body.

The following measurement procedure was used. 1. The patient was asked for the symptoms and illnesses, he/she suffers from. 2. About $20-40$ homeopathic drugs were chosen to the preliminary test according to the indications to the reported syndromes. 3. Next, 1 or 2 most strongly resonating drugs were chosen to the final test. 4. The homeopathic drug was administrated for the patient according to drug indications. The measurement of the resonance strength was performed about $1 \mathrm{~min}$ after every one globule administrated. 10 globuli were administrated maximally, altogether. The test was earlier broken, if the overdose resonance higher than $\mathrm{I}=5$ occurred earlier.

The preliminary results of 13 patients tested show that the resonance decreases initially after every globule administrated.

The approximately logarithmic decrease in amplification power was observed. Every globule decreased the maximal amplification about 4 - times. The lack of resonance was found after $2-5$ globuli administrated (in most cases - after 3 globuli). The further administration of -6 globuli did no change significantly the saturation state. Next, the resonance started to increase after further administration, pointing to the overdose effect. The logarithmic scaling was also observed in the overdose range, however, the multiplication factor was lower. In some cases, the measurement was repeated after $5-15 \mathrm{~min}$, showing often the vanishing of the overdosing resonance. This effect can suggest that the therapy should follow in the home therapy.

Conclusions: The results of the performed tests correlate with the homeopathic rule "SSC". MORA$\mathrm{kHz}$-resonance is probably a useful method for looking for the drugs that are necessary for the given patient. The correlation between the resonance and clinical therapeutic effect must be measured in order to confirm the usefulness of the presented method.

\section{FRACTALITY\&NONLOCALITY}

\section{Molski}

\section{Theoretical Chemistry Department A Mickiewicz University of Poznan ul. Grunwaldzka 6, 60 - 780 Poznan, Poland}

According to the Mandelbrot theory, the fractals are objects endowed with fractional (not integer) dimension and structural elements interrelated by the self-similar transformation. In the famous book "Fractal physiology" [1] the authors pointed out an interesting property of the fractal formation "....the fractal dimension remains approximately constant over many different scales. How can that be? How can 
different physical processes acting at different scales self-organize into fractal patterns. The mechanisms that link processes at different scales are mysterious. We make only a few tentative and speculative remarks. The first possibility is that something is shared across scales that cause them to adjust together. It must be something conserved (so that its balance is equalized across scales) and something minimized or maximized (so that it can be optimized at different scales). Thus, it must be something like free energy or entropy, and yet it is clearly not the free energy or entropy...." The lecture presents the theory of biological growth in the space-time with temporal fractal dimension [2 - 4], which indicates that this something can be identified with the quasi-quantum nonlocal spatial coherence, which interrelates at-thedistance all spatially separated sub - elements of the system providing the cooperative and self-organizing mechanisms responsible for the fractal creation on all scales of the matter organization. In this way different bio - processes over different spatial and temporal scales can organize into structures with a consistent overall spatial fractal dimension or changing globally in time the temporal fractal dimension.

[1] Bassingthwaighte J. B., Liebovitch L.S., West B. J. (1994) Fractal physiology. Oxford University Press, New York.

[2] Molski M., Konarski J. (2006) Neuronal differentiation and synapse formation in the space-time with temporal fractal dimension. Synapse, 60:567 - 572 .

[3] Molski M., Konarski J. (2008) Tumor growth in the space-time with temporal fractal dimension. Chaos, Solitons \& Fractals 36:811 - 818.

[4] Molski M. Fractal Time of Life. Lambert Academic Publishing 2012.

\section{FRACTALS\&ART}

\section{Molski, B. Czapiewska - Oleksa, J. Molski}

Theoretical Chemistry Department A Mickiewicz University of Poznan ul. Grunwaldzka 6, 60-780 Poznan, Poland

"Fractals \& Art" is a multimedial presentation on the Mandelbrot theory of fractal objects found in nature, generated by computer and created by artists. In particular, we will consider the mathematical objects of fractional dimension: Cantor set, Sierpinski carpet, Menger sponge as well as complex numbers and the self - similar transformation applied to create fractals. Fractal analysis of Jackson Pollock (1912 - 1956) paintings and the fractal interpretation of the Maurits Cornelis Escher (1898 - 1972) works will be carried out. In the final part the fractal movies created by making use of the of computer graphics techniques will be also demonstrated. The presentation is a joint project of scientist, artist Beata Czapiewska-Oleksa and computer graphics expert Julian Molski.

\section{IN SEARCH OF FACTORS INFLUENCING PROTEIN HANDEDNESS}

\author{
Iwona Mróz
}

Institute of Experimental Physics, University of Wroclaw,
Plac Maxa Borna 9, 50 - 204 Wroclaw, Poland
e-mail: imroz@ifd.uni.wroc.pl

Magnetic field is considered as a factor that may be useful to distinguish enantiomers, therefore the results of its interaction with chiral molecules have been widely investigated. It is also suggested that a magnetic field alone may lead to asymmetric synthesis if prochiral molecules are initially spatially oriented [1]. For molecules and molecular assemblies of biological importance, like proteins or protein complexes, their three-dimensional structures seem significant for protein-magnetic field interactions. Especially helical structures, including $\beta-$ helices containing $\mathrm{L}-$ as well as $\mathrm{D}$ - amino acids, may cause protein orientation in a magnetic field [e.g. $2-4$ ]. We review the experimental evidence of this problem.

To investigate if the handedness of biologically active proteins might partly result from primitive proteins' synthesis performed in the presence of a magnetic field, we analyze theoretically the handedness of sterically possible conformations of the terminal fragments of a simple helical protein synthesised in a magnetic field. A preliminary assumption that single long helical structures may be oriented in a static magnetic field is made [5], therefore the relative position of the analysed fragment and the direction of the magnetic field is controlled. To investigate the handedness of all possible conformations we assume that protein synthesis can occur from both protein termini and both amino acid stereoisomers can be included into the chain.

[1] Barron 1 L. D. (1994) Can magnetic field induce absolute asymmetric synthesis? Science 266:1491 - 1492.

[2] Arnesano F., Banci L., Bertini I., Van der Wetering K., Czisch M., Kaptein R. (2000) The autoorientation in high magnetic fields of oxidized cytochrome b562 as source of constrains for solution structure determination. $J$ Biomol NMR 17, $295-304$.

[3] Marcotte I., Bélanger A., Auger M. (2006) The orientation effect of gramicidin $\mathrm{A}$ and $\mathrm{Eu}+$-doped bicelles as studied by solid state NMR and FT-IR spectoscopy. Chem Phys Lipids 139:137 - 149.

[4] Numoto N., Schimizu K., Matsumoto K., Miki K, Kita A. (2013) Observation of the autoorientation of membrane protein crystals grown in high magnetic force field. $J$ Cryst Growth 367:53 - 56.

[5] Mróz I. (2005) Spatial separation of right- and lefthanded protein molecules in external magnetic field $\mathrm{s}-\mathrm{a}$ 
hypothesis. WSEAS Transactions on Biology and Biomadicine 2:381 - 385 .

\section{ELECTROMAGNETIC FIELDS AND WATER: THE POTENTIAL FOR NON- LOCALITY}

\author{
N. J. Murugan ${ }^{1,2}$, L. M. Karbowski ${ }^{1,2}$, \\ M. A. Persinger ${ }^{1,2}$
}

${ }^{1}$ Biomolecular Sciences Program, Laurentain University, Sudbury, P3E 2C6 Ontario

${ }^{2}$ Neuroscience Research Group, Laurentain University, Sudbury, P3E 2C6 Ontario

Whereas the function of matter is determined by its spatial organization, such as molecules, the function of electromagnetic fields is determined primarily by temporal patterns. Application of the appropriately patterned, weak magnetic field has been shown to dissolve planarian and to inhibit the growth of several different lines of cancer cells but not normal cells by affecting the plasma membrane. Living systems occur within an aqueous environment where viability is determined by narrow bands of temperature, $\mathrm{pH}$ and proportion of water. We present experimental evidence that specific, physiological patterns of weak magnetic fields that can potentially influence membrane magnetic moment of cells produce specific changes in $\mathrm{pH}$ within spring water. These exposures over several hours elicit discrete microshifts with durations of 20 to $40 \mathrm{~ms}$ in duration that are similar to those found within human cerebral cortices and reflects its $\sim 40 \mathrm{~Hz}$ coherent activity. The magnitudes of these shifts are consistent with a fundamental quantum of about $10^{-20} \mathrm{~J}$ which is the energy generated between the distance of the potassium ions that produce the membrane potential and the peak values for the action potential. Exposure to physiologically-patterned magnetic fields that imitate intrinsic configurations produces a shift in spectrophotometer profiles of about $10 \mathrm{~nm}$ or the width of a plasma membrane. These changes suggest that information can be represented within water along boundary conditions such as plasma cell membranes. Productions of specific magnetic fields, that would be required if photons exhibit a non-zero mass, in two different loci produce a non-locality or entanglement in both photon generation and shifts in $\mathrm{pH}$.

\section{POSTULATED PHYSICAL LINKS IN CANCER TRANSFORMATION}

\section{J. Pokorný}

Institute of Photonics and Electronics, ASCR, Prague, Czech Republic
Energy supply to a biological system excites and sustains a state far from thermodynamic equilibrium which is a basic condition for existence of life. Processes based on energy supply belong to the essentials for biological activity. Energy is transformed into physical forces and useful work for biological needs. But the principle processes and mechanisms of chemical energy transformation were not completely understood. Long range interactions and formation of mesoscopic and macroscopic structures indicate long range physical forces acting in biological systems. Distribution of charges in biological molecules and structures and exceptional electrical polarity of biological objects suggest electrodynamic and electromagnetic field generated in biological systems. Many authors published experimental results giving an account of electromagnetic activity in living systems. Generating function in the living cell was ascribed to microtubules in the cytoskeleton. Resonant frequencies of individual microtubules were recently investigated in a systematic fashion in the frequency range from $1 \mathrm{kHz}$ to $20 \mathrm{GHz}$. Two frequency bands were disclosed in the region below $1 \mathrm{GHz}$. One band has resonant frequencies $10-30 \mathrm{MHz}$, the other 100 $200 \mathrm{MHz}$. Oscillations in microtubules are possible under strict conditions in the cell: low damping by surrounding liquid medium, nonlinearity of vibrations, electric polarity, and sufficient energy supply. Mitochondria establish a strong static electric field resulting in ordered water around them which conditions low damping. The generated electromagnetic field may provide many working functions including synchronization and information transfer. The brain activity, central control function, instinct of self-preservation, and consciousness have been postulated to depend on electrodynamic processes, mainly of quantum nature. If the electrodynamic activity has any biological function then its disturbances have to result in pathological states. Pathological agents may attack biophysical processes directly or indirectly as a consequence of biochemical and genetic defects. The cancer transformation pathway contains a link of mitochondrial dysfunction, i.e. decreased oxidative metabolism. Therefore, cancer process is a pathological state disturbing energy processing system including generation of the electromagnetic field.

The experimental findings on biological electromagnetic activity represent a domain of singular items without adequate connections and sufficient experimental reproductions. On the other hand further experimental verifications need theoretical background. Basic items of biological electromagnetic activity were formulated in the form of postulates to set up a solid foundation for further theoretical and experimental studies in a new interdisciplinary field-physical biology and 
physiology. First postulates concern existence of endogenous coherent electromagnetic field, generating structure, and supporting organelles. Other postulates describe function of the electrodynamic field in biological cells and the brain. The last postulate concerns pathological state of the electrodynamic field in cancer development.

The postulates set up a solid foundation for further theoretical and experimental studies in a new interdisciplinary field. Cancer analysis is presented. Malignant links in cancer transformation are conditioned by dysfunction of mitochondria which can develop in cancer cells (the normal Warburg effect) or in fibroblasts associated with cancer cells (the reverse Warburg effect). In the normal or the reverse Warburg effect type of cancers the power of the electromagnetic field is predicted to be lower or higher than in healthy cells, respectively. In both cases the predicted frequency spectra are rebuilt and shifted in direction corresponding to the increased or decreased power. Interaction between cancer and healthy cells is disturbed and a pathway for local invasion and metastasis is open. An overview of experimental results will be presented.

\section{NONLINEAR OPTICAL INTERACTIONS WITH A PROTEIN COMPLEX}

\section{V. G. L. N. Rao, Ch. Yelleswarpu}

Department of Physics, University of Massachusetts Boston, 100 Morrissey Blvd, Boston, MA 02125

Our group has been working on basic nonlinear optics of the protein complex Bacteriorhodopsin(bR) thin films with low power cw lasers. The unique feature of this biomaterial is its flexibility. Absorption of a visible photon by $\mathrm{bR}$ triggers the photocycle, starting from the initial $\mathrm{B}$ state to the relatively long lived $\mathrm{M}$ state via short lived intermediate states. Molecules in the $\mathrm{M}$ state can revert to the initial B state thermally in milliseconds via short lived intermediate states or they can go back directly to B state within nanoseconds by shining blue light. Both life times can be altered by orders of magnitude using chemical methods or genetic mutation. The process of switching between $\mathrm{B}$ and $M$ states (chemical isomers) can go in both directions depending on wavelength, intensity and polarization of the incident light offering a variety of possibilities for manipulating amplitude, phase and polarization of the incident light. Over the years we studied the basic nonlinear optics- four wave mixing, phase conjugation, photo induced anisotropy etc. We successfully exploited the unique properties for many applications- all optical fast switching, modulation, computing, information processing, power limiting for laser eye protection, medical image processing, transient Fourier holography etc. More recently we are focusing on optical Fourier techniques for early detection of microcalcifications in mammograms for breast cancer diagnostics. We also developed an innovative technique of Fourier phase contrast microscopy and multimodal optical microscopy for live cell imaging of biological samples. I will present some highlights of our work with particular reference to development of inexpensive biomedical devices.

\section{A QUANTUM MECHANICAL INVESTIGATION OF TRAPPING OF IONS IN THE SELECTIVITY FILTERS OF KesA ION CHANNELS}

\section{V.Salari ${ }^{1}$, M. Sajjadi ${ }^{2}$}

${ }^{1}$ Department of Physics, Isfahan University of Technology, Iran

${ }^{2}$ Department of Physics, University of Shahrkord, Iran

Since the discovery of potassium $\mathrm{K}+$ channels, a surge of interest among investigators has been aroused to understand and explain their fascinating operational mechanisms. These protein complexes that are assembled by several proteins creating circular pores through the membrane operate in a fast and precise manner. The selectivity filter is believed to be responsible for the selection and fast conduction of particular ions across the membrane of an excitable cell. Other (generally larger) parts of the molecule such as the pore - domain gate control the access of ions to the channel protein.

Recently the occurrence of quantum coherence in the selectivity filter backbone is proposed by arguing that the backbone structure is not rigid as expected in classical models and vibrational excitations in $\mathrm{K}+$ ionchannels are the candidates to produce such coherence. On the other side, we already investigated the interaction of a single potassium ion within the surrounding carbonyl dipoles by analyzing solutions of the Schrödinger equation for the bacterial KcsA ion - channel. We showed that alkali ions can become highly delocalized in the filter region at warm temperatures. We claimed that a quantum mechanical calculation is needed to explain a fundamental biological property such as ion-selectivity in transmembrane ion - channels.

Here, we have used the Density Functional Theory (DFT) as a powerful ab initio method to derive a surface potential that $\mathrm{K}+$ feels inside the selectivity filter. The velocity and kinetic energy of ions as well as the carbonyl group's frequencies in different states have been obtained via the results of Gromacs software. To do these calculations we have used the entry $1 \mathrm{~K} 4 \mathrm{C}$ from the Protein Data Bank to represent the potassium channel. An analogy between classical and quantum mechanical models is discussed. It will be shown that quantum effects have interesting and significant properties in the selectivity filter and ion channel. For example, ions are trapped at specific locations in the filter and changed to very cold atoms 
which can interfere with adjacent ions causing efficiently functioning of the selectivity filter.

\section{NONLINEAR IONIC PULSES ALONG CYTOSKEKETAL MICROTUBULS}

\author{
M. V. Satarić
}

\author{
Faculty of Technical Sciences, University of Novi Sad \\ Trg D. Obradovića 6, 21000 Novi Sad, Serbia \\ e-mail bomisat@neobee.net
}

Microtubules (MTs) are cytoskeletal biopolymers shaped as nanotubes that are essential for cell motility, cell division and intracellular trafficking. Here we investigate their polyelectrolyte character that plays a very important role in ionic transport throughout cellular environment. The model we propose demonstrates an essentially nonlinear behavior of ionic currents which are guided by MTs. These features are primarly due to the dynamics of tubulin's C - terminal tails which are extended out of the surface of the MT cylinder $[1,2]$. We also demonstrate that the origin of nonlinearity stems from the nonlinear capacitance of each tubulin dimer. This brings about conditions required for the creation and propagation of solitonic ionic waves along the MT axis. We conclude that a MT plays the role of a biological nonlinear transmission line for ionic currents [2]. These currents might be of interest for cell division and perhaps can play some important role even in cognitive processes in nerve cell.

We expect that this kind of localized ionic waves could have the basic role in many vital cellular activities.

First the process of cell division needs the synchronized depolymerisation of MTs. It can be caused by the localized $\mathrm{Ca}^{++}$waves of our model, reaching MT plus ends and triggering the onset of massive detachment of tubulin dimers from MT tips. If just pure diffusion of $\mathrm{Ca}^{++}$ions through the bulk cytosol is left to rule this depolymerisation, the mistakes in this process would overwhelm the needed coordination.

In the very interesting paper [3] it was explained that in hair bundles (kinocilium) in the inner ear, consisting of the MT doublets, the myosin motordriven oscillations are controlled by $\mathrm{Ca}^{++}$ions directed from ion channels along MTs.

These ions cause a fraction of myosin motors to detach and thus tune the oscillations of kinocilium as the reaction on a corresponding acoustic signal. This mechanism can also be explained by the localized $\mathrm{Ca}^{++}$waves elaborated here.

[1] Satarić M. V., Ilić D., Ralević N. and Tuszynski J. A. (2009) Eur. Biophys. J. 38: 637.

[2] Satarić M. V., Sekulić D. and Živanov M. (2010) Journ. Comput. Theor. Nanosci. 7, 2281.
[3] Duke T. (2003) J. Phys. Condensed Matter 15, S1747.

\section{THE INFLUENCE OF UV-IRRADIATION WITH THE WAVELENGTH $254 \mathrm{~nm}$ ON HUMAN HAIR}

\author{
A. Sionkowska, B. Kaczmarek
}

Faculty of Chemistry, Nicolaus Copernicus University, Gagarin 7, 87 - 100 Torun, Poland

Human and animal hairs are exposed to sun radiation during lifetime and hence it is necessary to study the properties of hair after UV - irradiation, which can be a part of sunlight. The influence of UV radiation with the wavelength $254 \mathrm{~nm}$ on virgin curly brown hair was studied. Samples of hair were irradiated for 8 and 16 hours and the results of mechanical properties and visual appearance were compared with virgin hair. Air - dried hair was submitted to treatment with UV irradiation. Scanning electron microscopy images were made to show changes in the structure of hair. The mechanical properties of human hair before and after UV-irradiation were investigated by mechanical testing. The changes in such mechanical properties as ultimate tensile strength and percentage of elongation were investigated. Moreover, the Young's modulus was calculated based on the above measurements. The results have shown hat the mechanical properties of hair were greatly affected by the time of UV irradiation. Ultimate tensile strength and ultimate percentage of elongation decreased after UV irradiation of hair. Increasing UV-irradiation led to a decrease in Young's modulus of human hair. The mechanism of photodegradation of human hair will be discussed.

\section{A MODEL FOR INTEGRATIVE BIOPHYSICAL MEDICINE- IN PRACTICE, RESEARCH AND EDUCATION}

\section{G. G. Sullivan}

We are entering a new phase of understanding of the biological system. Following decades of research, we now possess indisputable evidence of an aspect of biology never before given serious consideration in everyday traditional medicine. I'd like to present a model based on this perspective: Integrative Biophysical Medicine in practice, research and education. Enter a new dimension, the interface by which all medicine should from henceforth be evaluated, namely, how effectively a given therapy can strengthen one's "buoyancy," i.e. the stored structural energy of excitation, providing for one's potential energy, which is in turn used to promote work performance, and maintenance of one's vitality 
and viability in the environment.

In practice, to maintain resiliency, structural and functional integrity, and processing of environmental inputs as they arise, the body needs energy: again, electrons and photon energy of excitation, providing ammunition for metabolism broadly. We have a direct, measurable pathway through our mitochondria to support this energetic density, by extracting the light and electrons from our food, and delivering them to oxygen and the body water, ultimately converting the energy to a receptive and usable form. As presented previously (EDALC 2011: Mitochondrial disruption in cancer--clinical assessment and support), we have already developed "the metabolic tune-up." Starting with assessment and support of the essential elements--the placeholders, the catalysts and cofactors, the structure by which to capture light and electrons, we must then assure the "basic process" as described by Erwin Bauer is in motion. As we maintain the living structure, energized, coordinated and supported by light-filled water domains, we give rise to coherence, reduction-oxidation interplay, autonomic nervous system balance, ability to ebb and flow, give and take, build and concentrate, and again, target internal buoyancy as the goal. The clinical question becomes "how strongly can we support one's energetic constitution?"

In research, of particular need is expansion of methods to capture and confirm the underlying energetic density and interplay, already alluded to in so many measurable ways. Help is needed to answer the increasing numbers of questions arising from the traditional research infrastructure, all collaborating our energetic model. Examples from fields including cancer research and embryology will be briefly presented, along with some of the measurement methods currently in use. Ideas for future directions of research and needs will be further outlined.

Finally, in education, we need to pilot "The Lecture Series" for Integrative Biophysical Medicine. As previously recognized, little exists in traditional lifescience education by way of an organized, complete overview of our energetic frame of reference. A proposed curriculum will be briefly outlined in hopes for feedback to facilitate the paradigm shift now unquestionably upon us.

Whole universities and research foundations need now to initiate concerted investigation. We need help. The time is now. A tangible organized model with which to move forward in medicine is upon us. 